\title{
Development of a new method to determine dynamic tensile strength of ballistic yarns
}

\author{
J. Nussbaum ${ }^{1, a}$, N. Faderl ${ }^{1}$, R. Nuesing ${ }^{1}$, C. Ha-Minh ${ }^{2}$, and F. Boussu ${ }^{2}$ \\ ${ }^{1}$ ISL, French-German Research Institute of Saint-Louis, 5 rue du General Cassagnou, BP. 70034, \\ 68301 Saint-Louis Cedex, France \\ ${ }^{2}$ ENSAIT - GEMTEX, 2 allée Louise et Victor Champier, BP. 30329, 59056 Roubaix Cedex, France
}

\begin{abstract}
Since the design of new soft protections is getting intensive, the study of the dynamic behavior of ballistic yarns becomes more and more important. This paper deals with the development of a new experimental method in order to obtain some dynamic parameters by performing dynamic tensile tests. The concept is quite simple: two projectiles, linked together by the ballistic yarn we want to study, are accelerated together with the help of a gas gun. The first one flies freely while the second one is suddenly stopped. The yarn is then loaded in tensile. Several measurements are performed during each test with the help of a high speed camera. First results on a polyethylene yarn are presented here and several improvements are proposed in order to improve the accuracy of the results.
\end{abstract}

\section{Introduction}

For the design of new ballistic protections, numerical simulations are required in order to save time and material as well as to understand the different mechanical phenomena that can occur during an impact. One of the main problems is to use realistic parameters as inputs for the material models implemented in the hydrodynamic codes. While these parameters are relatively easy to determine in the quasi-static regime, they remain generally unknown at the high strain rates involved in ballistic impact loadings. In the last decades, some experimental methods such as Taylor impact or Hopkinson bar tests have been developed in order to evaluate some parameters. Hard materials can thus be partially characterized with these kinds of tests but it is still a challenge to gather information on the dynamic behavior of soft materials such as fabrics due to the lack of adapted experiments.

Independently of the architecture of the fabric one wants to study, the basic element remains the yarn and has to be correctly modeled in order to obtain a realistic behavior of the whole woven structure. The work presented here is thus focused on the study of the tensile loading of ballistic yarns through the development of a new dynamic tensile test, still in progress. Other authors have published contributions on the influence of the strain rate on the mechanical properties of the yarn [1-4], but our approach is innovative. The corresponding experimental setup results from a step-by-step process where each step corresponds to the improvements of some parts of the setup and is presented in the second section. For this preliminary study, a Spectra 900 polyethylene fiber is used. Physical properties given by the provider are synthesized in the table 1. The first experimental results are then detailed in the third section. Because the numerical tools have not yet developed, we present in the forth section only a brief analysis of the results and more particularly some comments on their coherence. We conclude this paper by

a e-mail: julien.nussbaum@isl.eu proposing some improvements that will be applied in the future works.

\section{Experimental setup}

The idea is to develop a simple tensile test method that can achieve higher strain rates than usually encountered by classical tensile tests. For this purpose, we adapt the concept of sabot and projectile: we design two projectiles of two different diameters which can be combined together such as a projectile and a sabot. These two parts are linked by the polyethylene yarn, which is wound around two axes, one in each part. The technical drawing of the set is presented in the figure 1 while a picture of the opened system after a test can be seen on the figure 2 . In order to make the link with previous explanations, the projectile is referenced as 1 on the figure 1 , the sabot as 2 , and their axes respectively as 4 and 3 .

The system is then accelerated with the help of a $15 \mathrm{~mm}$ caliber gas gun. An additional mechanical piece is screwed at the muzzle of the gun, with a reduction of caliber. The largest part corresponding to the sabot is then stopped while the projectile continues to travel freely, making the yarn unwinding. After some milliseconds, the yarn is totally unwound and a tensile stress is applied to the yarn resulting in a resistive force that decelerates the projectile. If the stress becomes too large, the yarn breaks and the projectile goes on with a slower residual velocity. In the other case, when the stress remains limited, the yarn does not break and the projectile is completely stopped.

The strength of one yarn is too low to be able to highlight the very slight difference between the initial and residual velocity. The yarn is wound one time around the axis in the sabot and then returns to the projectile, where it is tied to its axis. We have thus the tensile strength that is shared between the two yarns. All the following results and analysis are presented for this configuration. The axis diameters are made as large as possible $(3 \mathrm{~mm})$ in order to limit the shear stress around the axis. After using tungsten 
Table 1. Physical properties of Spectra 900 fibers.

\begin{tabular}{ll}
\hline properties & value \\
\hline density & $0.97 \mathrm{~g} / \mathrm{cc}$ \\
ult. tensile strength & $2590 \mathrm{MPa}$ \\
elasticity modulus & $117 \mathrm{GPa}$ \\
\hline
\end{tabular}
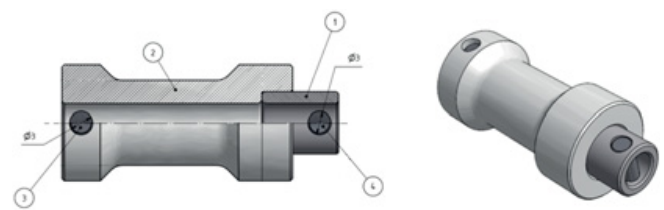

Fig. 1. Technical drawing of the set projectile + sabot (without yarn).

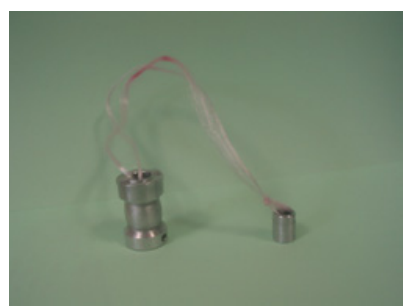

Fig. 2. Post-mortem projectile when the yarn did not break.

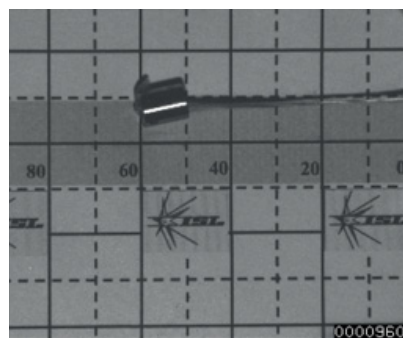

Fig. 3. High speed camera picture of a yarn during a test.

and steel, the two parts are now made of an aluminum alloy to decrease the total mass. The impulse transmitted to the yarn was indeed too high for the yarn which broke too soon to significantly decrease the velocity of the projectile. The total mass of the actual projectile is $1.2 \mathrm{~g}$ while the sabot weighs $9.4 \mathrm{~g}$. The knot remains a weak point in the yarn, because it can concentrate shear stress and then make the yarn breaking below the pure tensile stress limit. Some improvements are in progress and will be detailed in the last section.

Concerning the metrology, pictures of the complete experimental setup are presented in the figure 4. A scheme of the additional mechanical piece and the metrology associated, as well as an example of recordings are presented in figure 5. The initial velocity of the projectile is measured by two light barriers (1). A signal is then generated and triggers an high speed camera in order to record the flight of the projectile, to observe the behavior of the yarn and eventually to evaluate the residual velocity. The pictures are taken every $16 \mu$ s with a resolution of $320 \times 240$ pixels (an example is presented in the figure 3).

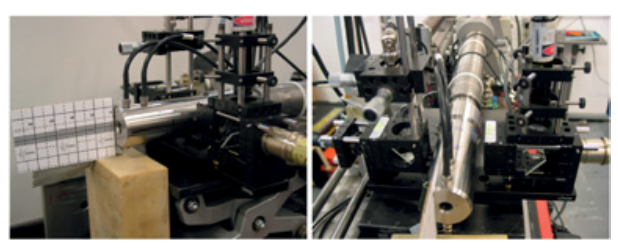

Fig. 4. General presentation of the experimental setup.

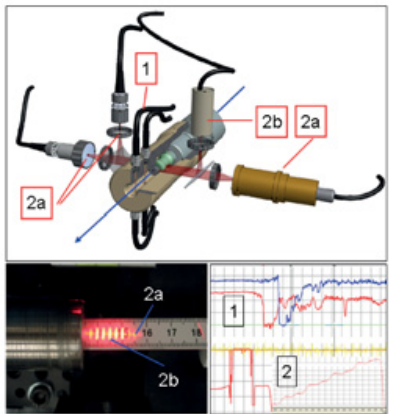

Fig. 5. Dual laser system for dynamic measurements.
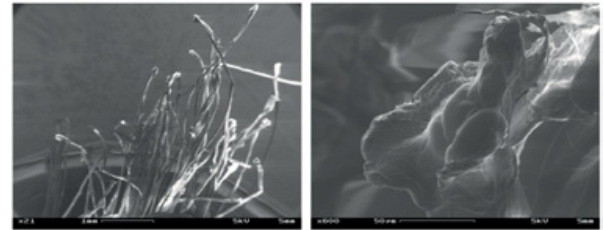

Fig. 6. Multifilament yarn after rupture (x21, left) - zoom on a filament (x500, right).

A dual laser system determines the position of the sabot while it is stopped in order to know where the two ends of the yarn are and thus to evaluate its length at each moment. The first laser (2a) delivers a small line with homogeneous (non-Gaussian) light intensity used to determine the motion of the support device. The second laser (2b) generates a line pattern (five lines) in order to deliver the relative local position of the support device. Both light signals are detected by two separate optosensitive devices. After each test, all the parts (sabot, projectile and yarn) are recovered and some post-mortem measurements are performed.

The measurements that are performed for each test are:

- initial length of the yarn;

- initial velocity of the projectile;

- final length of the sabot that has been crushed in the stopping phase;

- projectile position in function of time during its flight;

- position of the sabot during the impact at the muzzle;

- in some cases, the moment when the yarn breaks.

Of course, the relatively poor resolution of the high speed camera strongly limits the accuracy of the dynamic measurements. Some improvements are proposed in the last section. In addition, some Scanning Electron Microscopy (SEM) pictures of the rupture points on the yarns can be taken. Two pictures are presented in figure 6. 
Table 2. Experimental results on Spectra 900 fibers.

\begin{tabular}{clll}
\hline test \# & $L_{0}(\mathrm{~mm})$ & $V_{0}(\mathrm{~m} / \mathrm{s})$ & $V_{r}(\mathrm{~m} / \mathrm{s})$ \\
\hline 1 & 121.4 & 52.5 & 33.2 \\
2 & 117.3 & 55.3 & 37.8 \\
3 & 120.4 & 51.3 & 38.7 \\
4 & 120.3 & 50.7 & 31.5 \\
5 & 159.2 & 50.7 & 28.5 \\
6 & 160.3 & 52.1 & 27.5 \\
7 & 158.8 & 51.7 & 26.3 \\
8 & 158.3 & 50.8 & 25.7 \\
9 & 160.0 & 45.0 & 0.0 \\
10 & 159.1 & 44.5 & 0.0 \\
11 & 199.5 & 51.5 & 0.0 \\
12 & 197.0 & 56.3 & 0.0 \\
13 & 198.5 & 58.8 & 0.0 \\
14 & 197.5 & 64.1 & 30.6 \\
\hline
\end{tabular}

\section{First results}

A campaign of 14 tests has been performed with the Spectra 900 fiber with several goals:

1. to verify the reproducibility of the method;

2. to test the concept and the measurements accuracy;

3. to extract some trends about the dynamic behavior of the polyethylene yarn.

One can distinguish 3 series in the campaign, depending on the initial length of the yarn (length between the two axis): 120,160 and $200 \mathrm{~mm}$. For the first two lengths, the desired velocity is $50 \mathrm{~m} / \mathrm{s}$ and the tests are reproduced 4 times. Two additional tests have been performed with the $160 \mathrm{~mm}$ yarns at $45 \mathrm{~m} / \mathrm{s}$ in order to know if the yarn breaks or not. For the longest yarns, the velocity is increased from $50 \mathrm{~m} / \mathrm{s}$ (the yarn does not break) to $64 \mathrm{~m} / \mathrm{s}$ (the yarn breaks). The experimental results are synthesized in the table 2 . The initial length is noted $L_{0}$, the initial velocity $V_{0}$ and the residual velocity $V_{r}$.

\section{Analysis}

The analysis of the results is still delicate, as the accuracy of the high-speed camera is limited: an error of 1 pixel corresponds to a spatial error of $0.3 \mathrm{~mm}$. However, we can make some qualitative comments on the results by comparing the experimental results with the different lengths or velocities. First of all, results are relatively quite coherent, since the repetition of the tests \#1 and \#5 in similar conditions gives respectively similar results (excepted for the test \#3). That means that the knot is quite similar in all experiments, that was expected but not certain. Secondly, the yarn never breaks at the knot, but always at least ten millimeters away from the knot. The knot does not induce a weaker point than where the tensile strength becomes strong enough to have a rupture. By evaluating the moment and the length at which the yarn breaks, we can assume that this method enables us to reach strain rates higher than classical method $(\geq 100 / s)$.

Some tests were performed in order to evaluate the breaking velocity beyond which the yarn breaks. For a yarn

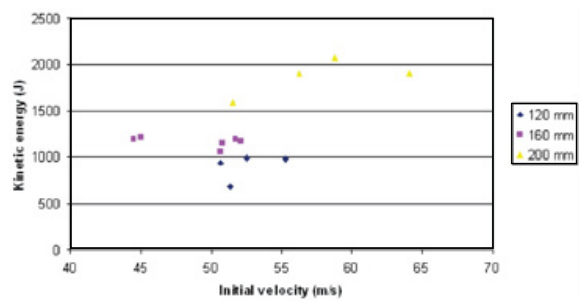

Fig. 7. Kinetic energy absorption in function of initial velocity and yarn length.

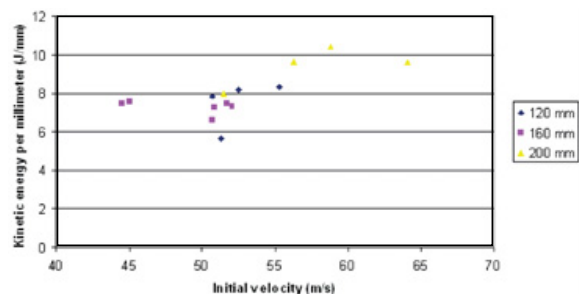

Fig. 8. Kinetic energy absorption per unit of length.

length of $160 \mathrm{~mm}$, we obtain a breaking velocity between $45 \mathrm{~m} / \mathrm{s}$ and $50 \mathrm{~m} / \mathrm{s}$ while for a yarn length of $200 \mathrm{~mm}$, this velocity lies between $59 \mathrm{~m} / \mathrm{s}$ and $64 \mathrm{~m} / \mathrm{s}$. This phenomenon is logical in the sense that the additional length shared the stress applied to the yarn and then reduce the local strain in the yarn. If we study the kinetic energy absorbed by each yarn, we obtain the chart of the figure 7 . In the range $50 \mathrm{~m} / \mathrm{s}$ - $55 \mathrm{~m} / \mathrm{s}$, we clearly see that the kinetic energy absorbed by the yarns is increasing with the yarn length.

By dividing the kinetic energy absorbed by the initial length of the yarn, we can see on the graph of the figure 8 that, for a similar initial velocity, the normalized kinetic energy absorbed is equivalent. For all these comments, complementary results would have been welcomed to study the trends on a broader range of velocities or lengths. They will be performed in a close future. In parallel, some improvements on the experimental setup will be done.

\section{Conclusion and future work}

In this paper, we presented a new experimental method to perform tensile tests on yarns at higher strain rates than classical ones. The first results are promising since their reproducibility has been demonstrated and they are in good agreement with the logic. The accuracy of the measurements has still to be increased in order to make sure analysis of the results, especially for the high speed camera pictures. However, some trends can already be observed about the kinetic energy that is absorbed by the yarns.

In the next step, the accuracy has to be improved by focusing the high speed camera on a smaller window and by increasing the framerate (up to $10^{6} \mathrm{fps}$ ). The other possibility to track the evolution of the projectile's velocity is to use radar or laser devices in order to have a continuous recording of the position. Some improvements can be performed on the clamping system: the knot does not disturb the test, but it is still a weak point in the system. Other clamping systems could be tested, inspired by the 
quasi-static tests facilities. However, the size of the system implies very small mechanical pieces that are very difficult to produce, but the challenge is very interesting.

Some complementary tests on aramid fibers have been performed but not presented here. The first analyses about the dynamic behavior of this fiber have been done in [5]. The dynamic parameters (tensile strength) deduced from the experiments were used as input parameters in a numerical code and the computed results were close to the experimental ones. The improvement of the computed results was obvious compared to the ones with quasi-static input data. Numerical tools still have to be developed in order to perform more finer and systematic analysis for each tests.

\section{References}

1. Y. Wang and Y. Xia, Composites Part A 29A, (1998) 1411-1415

2. B. Gu, Composites Part B 34, (2003) 361-371

3. V.B.C. Tan, X.S. Zeng and V.P.W. Shim, International Journal of Impact Engineering 35, (2008) 1303-1313

4. M. Cheng, W. Chen and T. Weerasooriya, Journal of Engineering Materials and Technology 127, (2005) 197-203

5. C. HA-MINH, Comportement mecanique des materiaux tisses soumis a un impact balistique : approches experimentale, numerique et analytique ( $\mathrm{PhD}$ thesis, University of Lille 1, France), 2011 